\title{
Supporting information for: \\ Phosphate Polymer Nanogel for Selective and Efficient Rare Earth Element Recovery
}

Yilin Zhang ${ }^{a, b}$, Jiajun Yan ${ }^{c}$, Jiang Xu ${ }^{a, b}$, Chong Tian ${ }^{a, b}$, Krzysztof Matyjaszewski $^{c}$, Robert D. Tilton ${ }^{b, d, e}$ and Gregory V. Lowry ${ }^{a, b *}$

a. Department of Civil and Environmental Engineering, b. Center for Environmental Implications of Nano Technology (CEINT), c. Department of Chemistry, d. Department of Chemical Engineering and e. Department of Biomedical Engineering Carnegie Mellon University, Pittsburgh, Pennsylvania 15213, United States.

* Corresponding author: Phone: (412) 268-2948; Fax: (412) 268-7813;

E-mail address: glowry@cmu.edu (G.V. Lowry)

Number of Figures: 6

Number of Tables: 8

13 pages 
Table of Contents

Scheme S1. Schematic illustration for synthesis of PPN by inverse mini-emulsion polymerization.

Figure S1. UV-Vis absorbance spectra for $0.5 \mathrm{~g} \mathrm{~L}^{-1} \mathrm{PPN}$ before and after filtered with 0.45 $\mu m$ PTFE filter. The optical density of filtered sample is close to MilliQ water, indicating complete removal of PPN by filtration.

Figure S2. (a) Apparent zeta potential of PPN at $100 \mathrm{mg} \mathrm{L}^{-1}$ PPN concentration at pH 1, 3, 5 and 7. (b) Intensity-weighted hydrodynamic diameter distribution of PPN at $100 \mathrm{mg} \mathrm{L}^{-1}$ polymer concentration in water at $\mathbf{p H}=6.9$.

Figure S3. Sorption kinetic of REE by PPN using $10 \mathrm{mg} \mathrm{L}^{-1}$ each of Nd, Gd and Ho at pH 5.0 with $0.5 \mathrm{~g} \mathrm{~L}^{-1}$ PPN.

Figure S4. Fraction of REEs (Nd, Gd and Ho) dissolved at various pH values (a) at $100 \mathrm{mg}$ $\mathrm{L}^{-1}$ and (b) $100 \mu \mathrm{g} \mathrm{L}^{-1} \mathrm{REE}$ ion concentrations modeled by Visual Minteq.

Figure S5. Fraction of dissolved competing ions $\mathrm{Al}^{3+}(\mathrm{a}), \mathrm{Fe}^{2+}(\mathrm{b}), \mathrm{Fe}^{3+}$ (c) at $100 \mathrm{mg} \mathrm{L}^{-1}$ under different $\mathrm{pH}$ values in the absence of sorbents modeled by Visual Minteq. ..................5

Table S1. Elemental analysis for $\mathrm{C}, \mathrm{H}, \mathrm{O}$ and $\mathrm{P}$ in the synthesized PPN. ................................5

Table S2. Fitting parameters in sorption isotherm of Nd, Gd and Ho by PPN fitted with Langmuir model.

Where $\underline{C e}$ is the equilibrium concentration of adsorbate $\left(\mathrm{mg} \mathrm{L}^{-1}\right)$, qe is the amount of metal adsorbed per gram of the adsorbent at equilibrium $\left(\mathrm{mg} \mathrm{g}^{-1}\right), Q m$ is the maximum coverage capacity $\left(\mathrm{mg} \mathrm{g}^{-1}\right)$ and $\underline{K L}$ is the Langmuir isotherm constant $\left(\mathrm{L} \mathrm{mg}^{-1}\right)$...................6

Table S3. REE Sorption performance of benchmark sorbent materials as shown in Figure 2d. All $K_{d}$ value picked in the following studies are acquired below pH 8 since most of

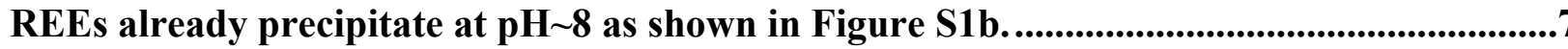

Table S4. Approximate average reported concentrations of selected cations and REEs in waste streams (ppm) including hydrothermal fluid, acid mine drainage, coal fly ash/ coal fly ash leachate and permanent magnet scrap. .................................................................

Table S6. REE concentrations in coal fly ash leachate at pH 5.0........................................10

Table S7. Competing ion concentrations in coal fly ash leachate at pH 5.0........................10

Table S8. Comparison of REE purity before and after extraction by various bench-mark materials. ................................................................................................................................................11

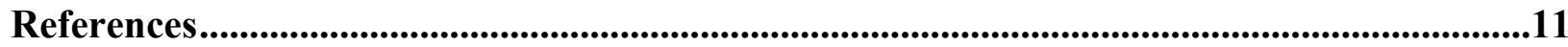




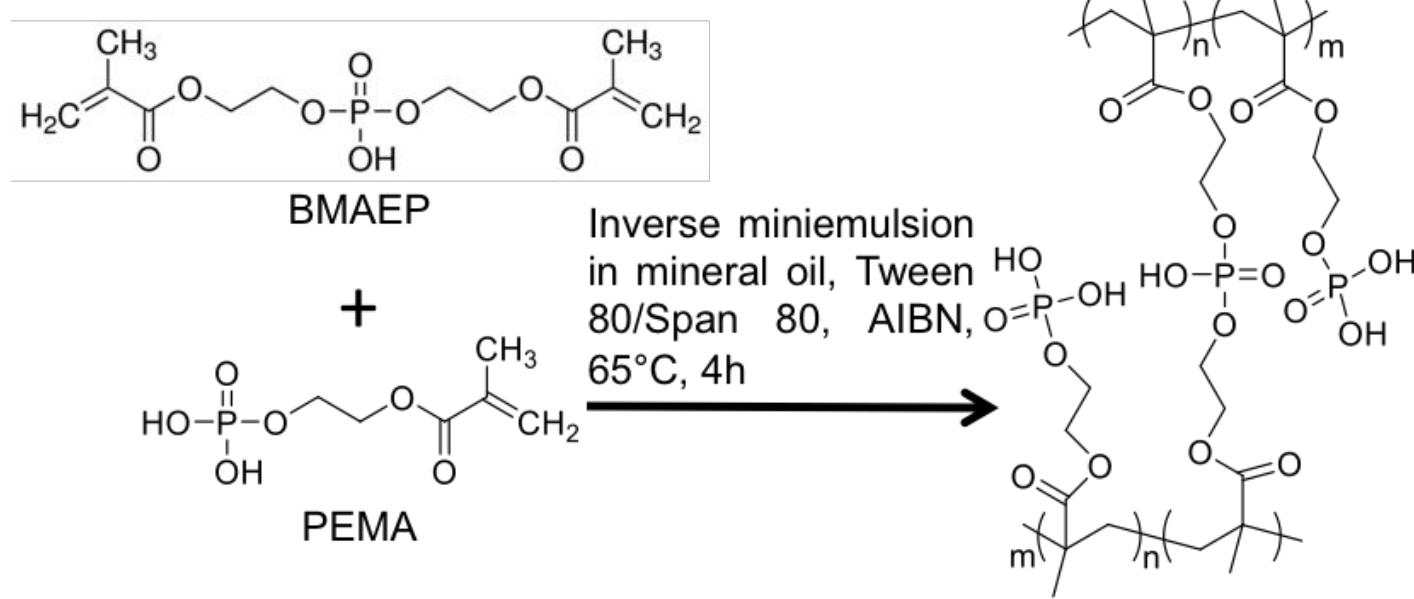

\section{Phosphate nanogel}

Scheme S1. Schematic illustration for synthesis of PPN by inverse mini-emulsion polymerization. Each BMAEP is a monodentate ligand, while each PEMA monomer is a bidentate ligand. The PEMA: BMAEP molar ratio is 3:1 in the PAEMAE used during PPN synthesis. So, among 5.2 mmol g-1 phosphorous in the PPN material, 3.9 mmol phosphate would be bidentate in PEMA and $1.3 \mathrm{mmol}$ would be monodentate phosphate in BMAEP. This provides total negative charge of 9.1 $\mathrm{mmol} \mathrm{g}^{-1}$. If all charges in PPN are available for capturing trivalent REEs, the PPN should be able to capture $\sim 3 \mathrm{mmol} \mathrm{g}^{-1}$ REEs.

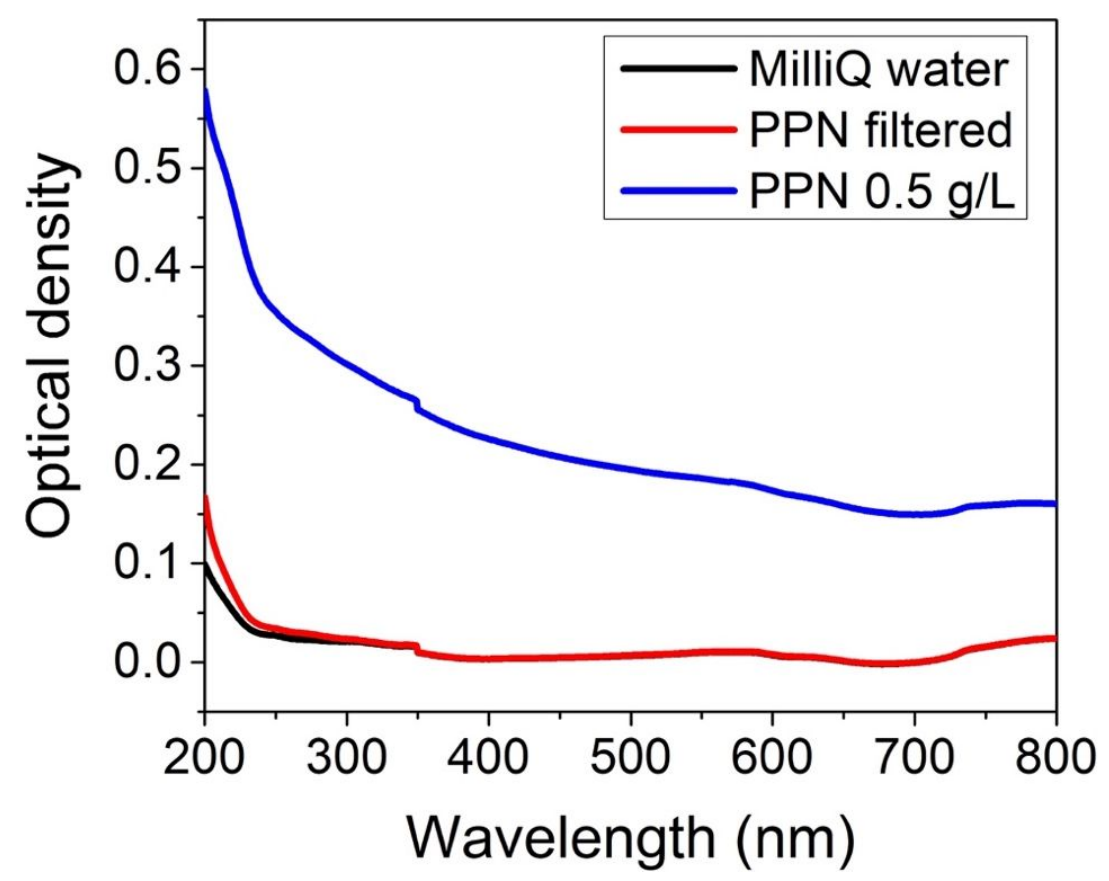

Figure S1. UV-Vis absorbance spectra for $0.5 \mathrm{~g} \mathrm{~L}^{-1} \mathrm{PPN}$ before and after filtered with $0.45 \mu \mathrm{m}$ PTFE filter. The optical density of filtered sample is close to MilliQ water, indicating complete removal of PPN by filtration. 

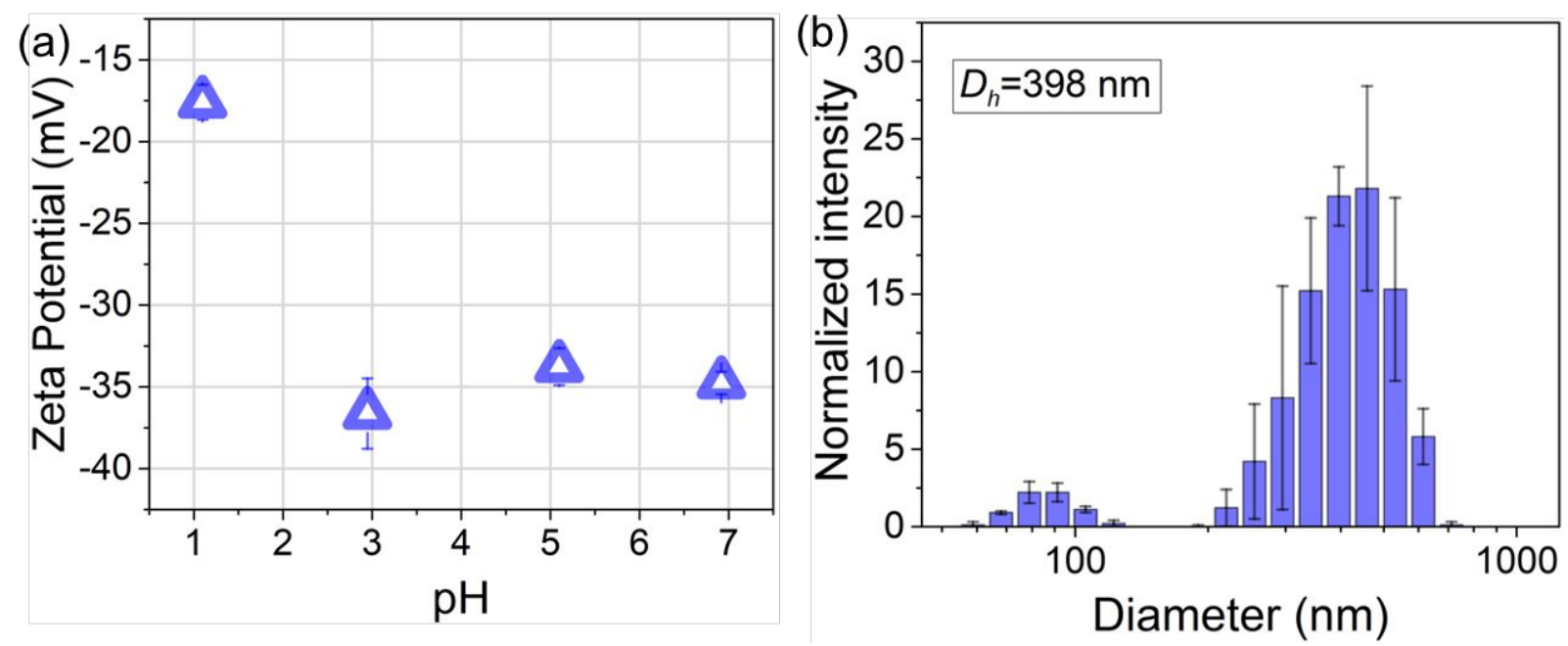

Figure S2. (a) Apparent zeta potential of PPN at $100 \mathrm{mg} \mathrm{L}^{-1} \mathrm{PPN}$ concentration at $\mathrm{pH} \mathrm{1,} \mathrm{3,} 5$ and 7. (b) Intensity-weighted hydrodynamic diameter distribution of PPN at $100 \mathrm{mg} \mathrm{L}^{-1}$ polymer concentration in water at $\mathrm{pH}=6.5$.

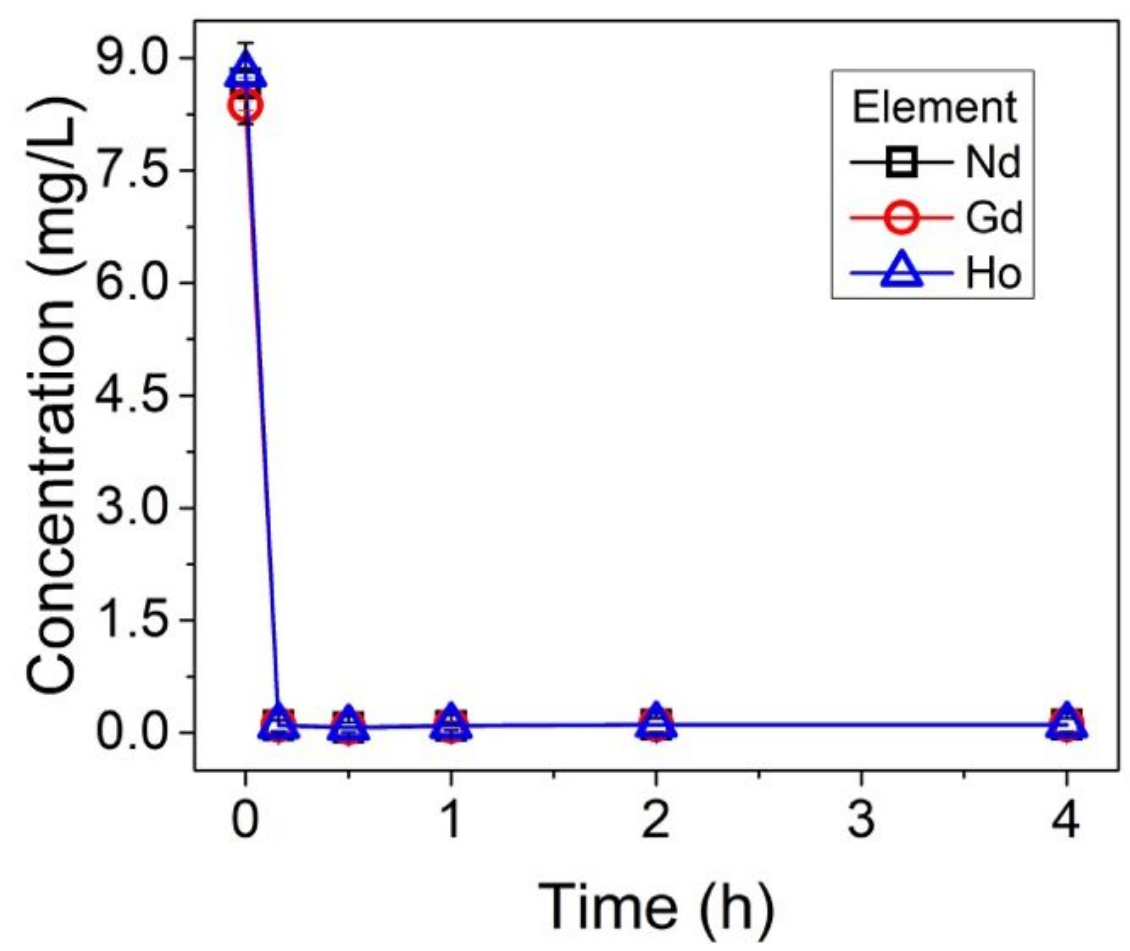

Figure S3. Sorption kinetic of REE by PPN using $10 \mathrm{mg} \mathrm{L}^{-1}$ each of $\mathrm{Nd}$, Gd and Ho at pH 5.0 with $0.5 \mathrm{~g} \mathrm{~L}^{-1}$ PPN. 

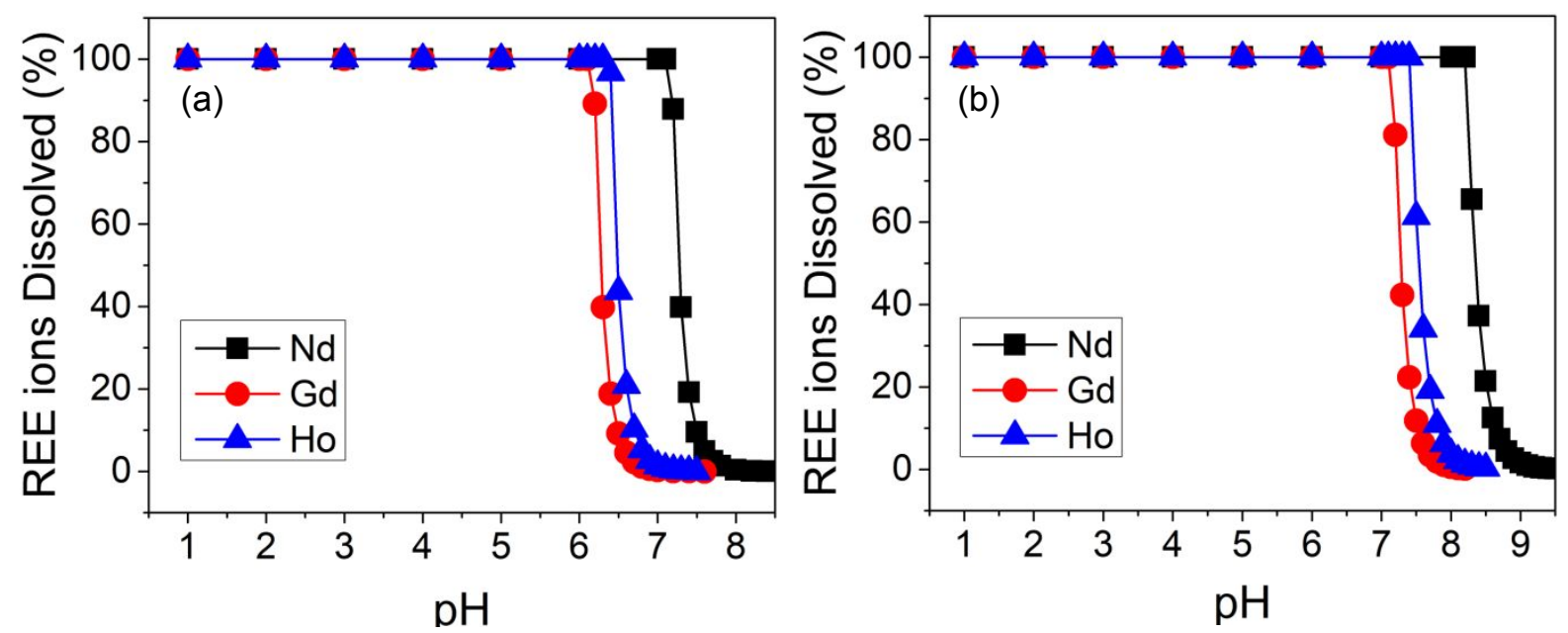

Figure S4. Fraction of REEs (Nd, Gd and Ho) dissolved at various $\mathrm{pH}$ values (a) at $100 \mathrm{mg} \mathrm{L}^{-1}$ and (b) $100 \mu \mathrm{g} \mathrm{L}^{-1} \mathrm{REE}$ ion concentrations modeled by Visual Minteq.
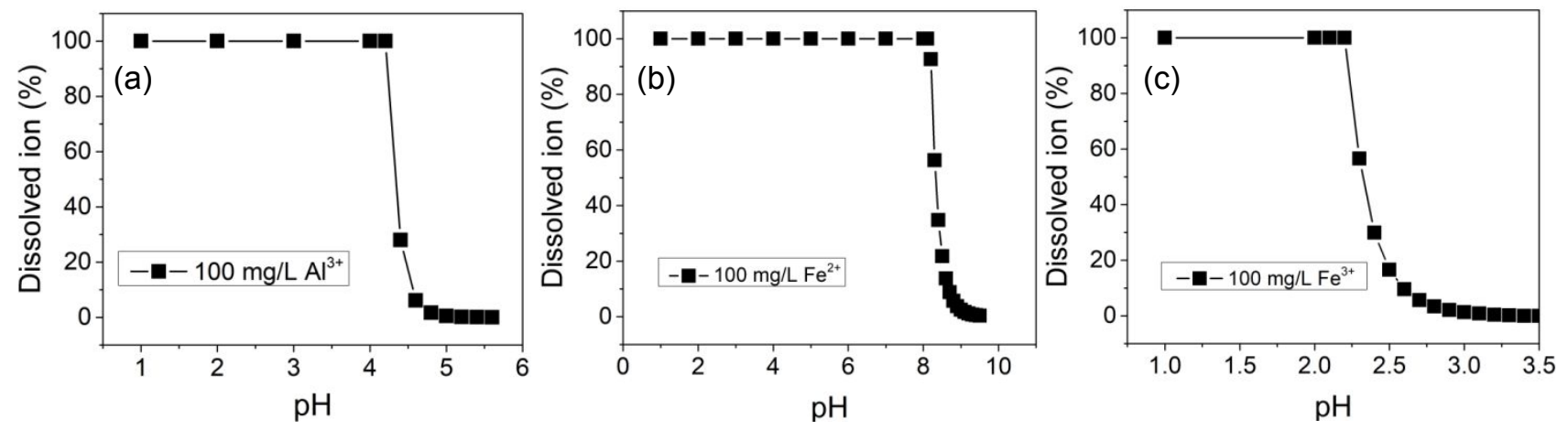

Figure S5. Fraction of dissolved competing ions $\mathrm{Al}^{3+}$ (a), $\mathrm{Fe}^{2+}(\mathrm{b}), \mathrm{Fe}^{3+}$ (c) at $100 \mathrm{mg} \mathrm{L}^{-1}$ under different $\mathrm{pH}$ values in the absence of sorbents modeled by Visual Minteq.

Table S1. Elemental analysis for C, H, O and P in the synthesized PPN.

\section{Elements Weight percent $(\%)$}

$\begin{array}{ll}\mathbf{C} & 30.0 \\ \mathbf{H} & 5.2 \\ \mathrm{O} & 49.6 \\ \mathrm{P} & 16.2\end{array}$


Table S2. Fitting parameters in sorption isotherm of Nd, Gd and Ho by PPN fitted with Langmuir model. The Langmuir isotherm is expressed by

$$
q_{e}=\frac{Q_{m} K_{L} C_{e}}{1+K_{L} C_{e}}
$$

Where $C_{e}$ is the equilibrium concentration of adsorbate $\left(\mathrm{mg} \mathrm{L}^{-1}\right), q_{e}$ is the amount of metal adsorbed per gram of the adsorbent at equilibrium $\left(\mathrm{mg} \mathrm{g}^{-1}\right), Q_{m}$ is the maximum coverage capacity $\left(\mathrm{mg} \mathrm{g}^{-1}\right)$ and $K_{L}$ is the Langmuir isotherm constant $\left(\mathrm{L} \mathrm{mg}^{-1}\right)$.

\begin{tabular}{|l|c|c|c|c|c|}
\hline Elements & $\mathbf{R}^{\mathbf{2}}$ & $\mathbf{Q}_{\mathbf{m}}\left(\mathbf{m g} \mathbf{g}^{-\mathbf{1}}\right)$ & $\mathbf{Q}_{\mathbf{m}} \mathbf{s t d}$ error & $\mathbf{K}_{\mathbf{L}}\left(\mathbf{L} \mathbf{~ m g}^{-\mathbf{1}}\right)$ & $\mathbf{K}_{\mathbf{L}} \mathbf{s t d}$ error \\
\hline Nd & 0.93 & 311 & 28.2 & 1.13 & 0.53 \\
\hline Gd & 0.90 & 316 & 37.5 & 1.38 & 0.62 \\
\hline Ho & 0.89 & 249 & 28.6 & 1.67 & 0.91 \\
\hline
\end{tabular}


Table S3. REE Sorption performance of benchmark sorbent materials as shown in Figure 2d. All $K_{\mathrm{d}}$ value picked in the following studies are acquired below $\mathrm{pH} 8$ since most of REEs already precipitate at $\mathrm{pH} \sim 8$ as shown in Figure $\mathbf{S 4 b}$.

\begin{tabular}{|c|c|c|c|c|c|c|c|c|c|}
\hline \multirow[b]{2}{*}{ Material } & \multirow[b]{2}{*}{$\begin{array}{c}\mathbf{q}_{\mathrm{e}} \\
(\mathbf{m g} \\
\left.\mathrm{g}^{-1}\right)\end{array}$} & \multirow[b]{2}{*}{$\begin{array}{c}K_{d} \\
(m L \\
g^{-}\end{array}$} & \multirow[b]{2}{*}{ Element } & \multicolumn{5}{|c|}{ Conditions in $K_{d}$ study } & \multirow[b]{2}{*}{$\begin{array}{c}\text { Sample } \\
\text { type }\end{array}$} \\
\hline & & & & $\mathrm{pH}$ & $\begin{array}{l}\mathrm{REE} \\
\text { conc } \\
(\mathrm{mg} \\
\left.\mathrm{L}^{-1}\right)\end{array}$ & $\begin{array}{c}\text { Sorbent } \\
\text { conc } \\
\left(\mathrm{mg} \mathrm{L} \mathrm{L}^{-}\right. \\
1)\end{array}$ & $\begin{array}{c}\mathrm{C}_{\mathrm{e}} \\
\left(\mathrm{mg} \mathrm{L}^{-1}\right)\end{array}$ & $\mathrm{KC}_{\mathrm{e}}$ & \\
\hline \multicolumn{10}{|c|}{ Included in Figure 2d } \\
\hline \multirow[t]{2}{*}{ PPN } & \multirow{2}{*}{316} & $3.1 \times 10^{5}$ & Ho & 7.0 & 0.1 & 100 & $3.1 \times 10^{-3}$ & $3.1 \times 10^{-3}$ & $\begin{array}{l}\text { Model } \\
\text { system }\end{array}$ \\
\hline & & $3.4 \times 10^{5}$ & $\mathrm{Nd}$ & 5.0 & 0.05 & 100 & $1.3 \times 10^{-3}$ & $1.4 \times 10^{-3}$ & $\begin{array}{l}\text { Fly ash } \\
\text { leachate }\end{array}$ \\
\hline $\begin{array}{l}\text { KIT-6- } \\
1,2-\text { PA }^{1}\end{array}$ & 8 & $5.0 \times 10^{4}$ & $\mathrm{Lu}$ & 4.0 & 0.03 & 2000 & $2.9 \times 10^{-4}$ & $1.8 \times 10^{-3}$ & $\begin{array}{l}\text { Model } \\
\text { system }\end{array}$ \\
\hline $\begin{array}{l}\text { TD- } \\
\text { DTPAD } \\
\mathrm{A}^{2}\end{array}$ & 1 & $1.0 \times 10^{4}$ & Gd & 3.0 & 0.1 & 10000 & $9.9 \times 10^{-4}$ & $1 \times 10^{-2}$ & $\begin{array}{l}\text { Model } \\
\text { system }\end{array}$ \\
\hline $\begin{array}{l}\text { BPG- } \\
\text { resin }\end{array}$ & 3.01 & $4.7 \times 10^{3}$ & $\begin{array}{l}\text { Nd, Gd, } \\
\text { Ho }\end{array}$ & 6.5 & 0.1 & 10000 & $2.1 \times 10^{-3}$ & $3.3 \times 10^{-3}$ & $\begin{array}{l}\text { Model } \\
\text { system }\end{array}$ \\
\hline $\begin{array}{l}\text { FJSM- } \\
\mathrm{SnS}^{4}\end{array}$ & 147 & $1.2 \times 10^{5}$ & $\mathrm{~Tb}$ & 5.9 & 3.96 & 1000 & 0.033 & 0.033 & $\begin{array}{l}\text { Model } \\
\text { system }\end{array}$ \\
\hline $\begin{array}{l}\text { PEI- } \\
\text { CNC3 }^{5}\end{array}$ & 106.4 & $2.5 \times 10^{5}$ & $\mathrm{Er}$ & 5.4 & 8 & 1000 & 0.032 & 0.081 & $\begin{array}{l}\text { Model } \\
\text { system }\end{array}$ \\
\hline $\begin{array}{l}\text { APTES- } \\
\text { C3-PAN } 6\end{array}$ & 120.7 & $1.2 \times 10^{5}$ & $\mathrm{La}$ & 7.0 & 25 & 1000 & 0.21 & 0.026 & $\begin{array}{l}\text { Model } \\
\text { system }\end{array}$ \\
\hline $\begin{array}{l}\text { C4mim@ } \\
\mathrm{UiO}-66^{7}\end{array}$ & 85 & $5.4 \times 10^{3}$ & Gd & 6.0 & 10 & 1000 & 1.56 & 0.10 & $\begin{array}{l}\text { Model } \\
\text { system }\end{array}$ \\
\hline \multicolumn{10}{|c|}{ Not included in Figure 2d due to higher KCe or lack of data for KCe calculation } \\
\hline $\begin{array}{l}\text { MIL- } \\
101- \\
\text { PMIDA }^{8}\end{array}$ & 90 & $7.1 \times 10^{3}$ & Gd & 6.0 & 20 & 1000 & 2.4 & 0.23 & $\begin{array}{l}\text { Model } \\
\text { system }\end{array}$ \\
\hline $\begin{array}{l}\text { LBT E- } \\
\text { coli }^{9}\end{array}$ & 28.3 & 31.6 & $\mathrm{~Tb}$ & 6.0 & 0.05 & $\begin{array}{c}10^{8} \\
\text { cell/mL }\end{array}$ & / & / & $\begin{array}{l}\text { Mine } \\
\text { tailing }\end{array}$ \\
\hline $\begin{array}{l}\text { UiO-66- } \\
\mathrm{COOH}- \\
\mathrm{ED}^{10}\end{array}$ & 79 & $3.6 \times 10^{3}$ & Gd & 6.0 & 40 & 1000 & 8.7 & 0.66 & $\begin{array}{l}\text { Model } \\
\text { system }\end{array}$ \\
\hline $\begin{array}{l}\text { PAA-S } \\
\text { HNFs }^{11}\end{array}$ & 269 & $7.7 \times 10^{4}$ & $\mathrm{Eu}$ & 6.0 & N.R. & 100 & I & I & $\begin{array}{l}\text { Model } \\
\text { system }\end{array}$ \\
\hline $\begin{array}{l}\text { PS- } \\
\text { DGAnf }^{12}\end{array}$ & 120 & $1.0 \times 10^{4}$ & $\mathrm{Nd}$ & 6.0 & 60 & 750 & 7.1 & 1.45 & $\begin{array}{l}\text { Model } \\
\text { system }\end{array}$ \\
\hline
\end{tabular}


Table S4. Approximate average reported concentrations of selected cations and REEs in waste streams (ppm) including hydrothermal fluid, acid mine drainage, coal fly ash/ coal fly ash leachate and permanent magnet scrap.

\begin{tabular}{|c|c|c|c|c|c|c|c|}
\hline Sample type & Study & $\mathbf{N a}$ & $\mathbf{C a}$ & Mg & Al & $\mathrm{Fe}$ & REEs \\
\hline \multirow{2}{*}{$\begin{array}{l}\text { Hydrothermal } \\
\text { Fluid }\end{array}$} & $\begin{array}{l}\text { Clark et al. } \\
(2010)^{15}\end{array}$ & 1600 & 200 & 70 & 10 & 20 & N.R. \\
\hline & $\begin{array}{l}\text { Maimoni } \\
(1982)^{16}\end{array}$ & 42000 & 18000 & 100 & $<1$ & 200 & N.R. \\
\hline $\begin{array}{l}\text { Acid mine } \\
\text { drainage }\end{array}$ & $\begin{array}{l}\text { Ayora et al. } \\
(2016)^{17}\end{array}$ & N.R. & 218 & 263 & 128 & 161 & 7.9 \\
\hline \multirow{2}{*}{$\begin{array}{c}\text { Coal fly } \\
\text { ash/leachate }\end{array}$} & $\begin{array}{l}\text { Liu et al. } \\
(2019)^{18}\end{array}$ & N.R. & 11430 & N.R. & 133400 & 83300 & 315.4 \\
\hline & $\begin{array}{l}\text { Smith et al. } \\
(2019)^{19}\end{array}$ & 681000 & 9210 & 7500 & 132000 & 19100 & 302 \\
\hline $\begin{array}{c}\text { Permanent } \\
\text { magnet scrap }\end{array}$ & $\begin{array}{l}\text { Mochizuki } \\
\text { et al. (2013) } \\
20\end{array}$ & 706000 & N.R. & N.R. & N.R. & N.R. & 263000 \\
\hline
\end{tabular}


Table S5. Information from different REE sorption studies for calculation of $\alpha(R E E / M e)$ in Figure 4.

PPN: phosphate polymer nanogel

MIL-101-PMIDA: chromium-based metal-organic framework with $N$-(phosphonomethyl) iminodiacetic acid.

UiO66-COOH-ED: UiO-66 metal-organic framework functionalized with - $\mathrm{COOH}$ and $-\mathrm{NH}_{2}$ groups.

C4mim@UiO-66: 1-butyl-3-methylimidazolium bromide entrapped UiO-66 metal-organic framework.

KIT-6-N-DGA: diglycolamide in covalently bound form.

BPG: N,N-bis(phosphonomethyl)glycine functionalized silica particle.

FJSM-SnS: $\left(\mathrm{Me}_{2} \mathrm{NH}_{2}\right)_{4 / 3}\left(\mathrm{Me}_{3} \mathrm{NH}\right)_{2 / 3} \mathrm{Sn}_{3} \mathrm{~S}_{7} \cdot 1.25 \mathrm{H}_{2} \mathrm{O}$.

APTES-C3-PAN: 3-Aminopropyl triethoxysilane-deacetylated chitin -2-naphthol.

\begin{tabular}{|c|c|c|c|c|c|c|}
\hline Material & $\begin{array}{l}K_{d} \text { with } \\
\text { excess } \\
\text { competing } \\
\text { ion }\left(\mathrm{mL} \mathrm{g}^{-1}\right)\end{array}$ & $\begin{array}{l}K_{d} \text { without } \\
\text { excess } \\
\text { competing } \\
\text { ion }\left(\mathrm{mL} \mathbf{g}^{-1}\right)\end{array}$ & $\begin{array}{l}\text { REE } \\
\text { concentration }\end{array}$ & $\begin{array}{l}\text { pH in } \\
\text { competing } \\
\text { sorption } \\
\text { study }\end{array}$ & $\begin{array}{l}\text { Competing ion } \\
\text { concentration }\end{array}$ & $\begin{array}{l}\text { Calculated } \\
\alpha(\mathrm{REE} / \mathrm{Me})\end{array}$ \\
\hline \multicolumn{7}{|c|}{ Included in selectivity study } \\
\hline \multirow[t]{2}{*}{ PPN } & $1.8 \times 10^{4}$ & \multirow{2}{*}{$2.8 \times 10^{5}$} & $\mathrm{Gd}, 100 \mu \mathrm{g} \mathrm{L}^{-1}$ & 3.0 & $\mathrm{Al}, 10 \mathrm{mg} \mathrm{L}^{-1}$ & 6.4 \\
\hline & $4.8 \times 10^{4}$ & & $\mathrm{Gd}, 100 \mu \mathrm{g} \mathrm{L}^{-1}$ & 3.0 & $\mathrm{Fe}, 3.4 \mathrm{mg} \mathrm{L}^{-1}$ & 5.8 \\
\hline $\begin{array}{l}\text { C4min@ } \\
\text { UiO66 }^{3}\end{array}$ & 4600 & 5400 & $\mathrm{Gd}, 40 \mathrm{mg} \mathrm{L}^{-1}$ & 6.0 & $\mathrm{Al}, 40 \mathrm{mg} \mathrm{L}^{-1}$ & 0.85 \\
\hline \multirow{2}{*}{$\begin{array}{l}\text { KIT-6-N- } \\
\text { DGA }^{4}\end{array}$} & 6500 & \multirow{2}{*}{6500} & $\mathrm{Gd}, 2 \mu \mathrm{g} \mathrm{L}^{-1}$ & 4.0 & $\mathrm{Al}, 2 \mu \mathrm{g} \mathrm{\textrm {L } ^ { - 1 }}$ & 1 \\
\hline & 6500 & & $\mathrm{Gd}, 2 \mu \mathrm{g} \mathrm{L}^{-1}$ & 4.0 & $\mathrm{Fe}, 2 \mu \mathrm{g} \mathrm{L}-1$ & 1 \\
\hline \multirow{2}{*}{$\mathrm{BPG}^{5,6}$} & 100 & \multirow{2}{*}{10000} & $\mathrm{Gd}, 100 \mu \mathrm{g} \mathrm{L}^{-1}$ & 2.0 & $\mathrm{Al}, 1000 \mathrm{mg} \mathrm{L}^{-1}$ & 100 \\
\hline & 122.2 & & $\mathrm{Gd}, 100 \mu \mathrm{g} \mathrm{L}^{-1}$ & 2.0 & $\mathrm{Fe}, 1000 \mathrm{mg} \mathrm{L}^{-1}$ & 120 \\
\hline \multirow{2}{*}{$\begin{array}{l}\text { KIT-6- } \\
1,2-\mathrm{PA}^{9}\end{array}$} & 66263 & \multirow{2}{*}{53541} & $\mathrm{Lu}, 1.23 \mu \mathrm{g} \mathrm{L}^{-1}$ & 4.0 & $\mathrm{Al}, 955 \mu \mathrm{g} \mathrm{L}^{-1}$ & 961 \\
\hline & 66263 & & $\mathrm{Lu}, 1.23 \mu \mathrm{g} \mathrm{L}^{-1}$ & 4.0 & $\mathrm{Fe}, 1.86 \mu \mathrm{g} \mathrm{L}^{-1}$ & 1.9 \\
\hline $\begin{array}{l}\text { FJSM- } \\
\mathrm{SnS}^{7}\end{array}$ & 5400 & $1.2 \times 10^{5}$ & $\mathrm{Eu}, 6.01 \mathrm{mg} \mathrm{L}^{-1}$ & 5.0 & $\mathrm{Al}, 118 \mathrm{mg} \mathrm{L}^{-1}$ & 0.92 \\
\hline $\begin{array}{l}\text { APTES- } \\
\text { C3-PAN }^{8}\end{array}$ & $1.3 \times 10^{4}$ & $1.32 \times 10^{5}$ & $\mathrm{Sc}, 25 \mathrm{mg} \mathrm{L}^{-1}$ & 4.0 & $\mathrm{Al}, 250 \mathrm{mg} \mathrm{L}^{-1}$ & 1.01 \\
\hline \multicolumn{7}{|c|}{ Not included in selectivity study } \\
\hline \multirow{2}{*}{$\begin{array}{l}\text { MIL-101- } \\
\text { PMIDA }^{1 \text { a }}\end{array}$} & 4500 & \multirow{2}{*}{7129} & $\mathrm{Gd}, 50 \mathrm{mg} \mathrm{L}^{-1}$ & 5.5 & $\mathrm{Al}, 50 \mathrm{mg} \mathrm{L}^{-1}$ & 0.63 \\
\hline & 4500 & & $\mathrm{Gd}, 50 \mathrm{mg} \mathrm{L}^{-1}$ & 5.5 & $\mathrm{Fe}, 50 \mathrm{mg} \mathrm{L}^{-1}$ & $0.63^{b}$ \\
\hline \multirow{2}{*}{$\begin{array}{l}\text { UiO66- } \\
\mathrm{COOH}- \\
\mathrm{ED}^{2} \mathrm{a}\end{array}$} & 3000 & \multirow{2}{*}{3600} & $\mathrm{Gd}, 40 \mathrm{mg} \mathrm{L}^{-1}$ & 6.0 & $\mathrm{Al}, 40 \mathrm{mg} \mathrm{L}^{-1}$ & 0.83 \\
\hline & 3000 & & $\mathrm{Gd}, 40 \mathrm{mg} \mathrm{L}^{-1}$ & 6.0 & $\mathrm{Fe}, 40 \mathrm{mg} \mathrm{L}^{-1}$ & $0.83^{b}$ \\
\hline $\begin{array}{l}\text { C4min@ } \\
\mathrm{UiO}^{3}{ }^{3}\end{array}$ & 4600 & 5400 & $\mathrm{Gd}, 40 \mathrm{mg} \mathrm{L}^{-1}$ & 6.0 & $\mathrm{Fe}, 40 \mathrm{mg} \mathrm{L}^{-1}$ & $0.85^{\mathrm{b}}$ \\
\hline $\begin{array}{l}\text { FJSM- } \\
\mathrm{SnS}^{7}\end{array}$ & 5400 & $1.2 \times 10^{5}$ & $\mathrm{Eu}, 6.01 \mathrm{mg} \mathrm{L}^{-1}$ & 5.0 & $\mathrm{Fe}, 53.2 \mathrm{mg} \mathrm{L}^{-1}$ & $0.4^{b}$ \\
\hline $\begin{array}{l}\text { APTES- } \\
\text { C3-PAN }^{8}\end{array}$ & $1.3 \times 10^{4}$ & $1.3 \times 10^{5}$ & $\mathrm{Sc}, 25 \mathrm{mg} \mathrm{L}^{-1}$ & 4.0 & $\mathrm{Fe}, 250 \mathrm{mg} \mathrm{L}^{-1}$ & $1.01^{\mathrm{b}}$ \\
\hline
\end{tabular}




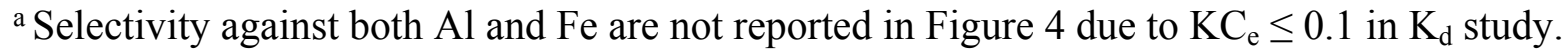

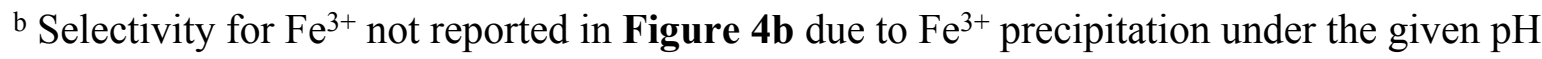
conditions for REE competitive sorption studies.

Table S6. REE concentrations in coal fly ash leachate at $\mathrm{pH}$ 5.0.

\begin{tabular}{|c|c|}
\hline Element & Concentration (ppb) \\
\hline Sc & $108.1 \pm 6.3$ \\
\hline Y & $84.4 \pm 0.9$ \\
\hline La & $123.5 \pm 1.4$ \\
\hline Ce & $106.1 \pm 1.2$ \\
\hline Pr & $14.3 \pm 0.1$ \\
\hline Nd & $48.4 \pm 0.5$ \\
\hline Sm & $10.5 \pm 0.3$ \\
\hline Eu & $27.8 \pm 0.5$ \\
\hline Gd & $14.2 \pm 0.5$ \\
\hline Tb & $1.7 \pm 0.1$ \\
\hline Dy & $4.4 \pm 0.1$ \\
\hline Ho & 0 \\
\hline Er & $2.1 \pm 0.03$ \\
\hline Tm & $0.31 \pm 0.01$ \\
\hline Yb & $2.3 \pm 0.1$ \\
\hline Lu & $0.64 \pm 0.07$ \\
\hline
\end{tabular}

Table S7. Competing ion concentrations in coal fly ash leachate at $\mathrm{pH} 5.0$.

\begin{tabular}{|c|c|}
\hline Element & Concentration (ppb) \\
\hline $\mathbf{M g}$ & $103971 \pm 1120$ \\
\hline $\mathbf{A l}$ & $2000.3 \pm 10.1$ \\
\hline $\mathbf{C r}$ & $41.8 \pm 2.5$ \\
\hline $\mathbf{F e}$ & $267.9 \pm 14.1$ \\
\hline $\mathbf{C o}$ & $138.3 \pm 2.3$ \\
\hline $\mathbf{N i}$ & $330.7 \pm 6.1$ \\
\hline $\mathbf{C u}$ & $902.5 \pm 38.3$ \\
\hline $\mathbf{Z n}$ & $497.4 \pm 4.4$ \\
\hline $\mathbf{P b}$ & $333.9 \pm 7.3$ \\
\hline $\mathbf{C d}$ & $7.2 \pm 0.7$ \\
\hline $\mathbf{B a}$ & $6091.9 \pm 76.4$ \\
\hline $\mathbf{T h}$ & $20.9 \pm 3.1$ \\
\hline $\mathbf{U}$ & $4.6 \pm 0.3$ \\
\hline $\mathbf{C a}$ & $6.9 \times 10^{*}$ \\
\hline \multicolumn{2}{|c}{} \\
\hline
\end{tabular}


*concentration of $\mathrm{Ca}$ in leachate calculated from $\mathrm{CaO}$ concentration in fly ash measured by $\mathrm{X}$ ray fluorescence $(\mathrm{XRF})$.

Table S8. Comparison of REE purity before and after extraction by various bench-mark materials.

\begin{tabular}{|c|c|c|c|c|}
\hline Material & REE feed stock & $\begin{array}{l}\text { REE purity in } \\
\text { raw material } \\
(\%)^{\mathrm{a}}\end{array}$ & $\begin{array}{l}\text { REE purity } \\
\text { after extraction } \\
(\%)^{\mathrm{a}}\end{array}$ & $\begin{array}{l}\text { REE } \\
\text { concentration } \\
\text { factor }^{b}\end{array}$ \\
\hline $\begin{array}{l}\text { PPN (this } \\
\text { study) }\end{array}$ & $\begin{array}{l}\text { Coal fly ash } \\
\text { leachate }\end{array}$ & 0.48 & 9.9 & 21 \\
\hline $\begin{array}{l}\text { Escherichia coli } \\
\text { microbe bead }^{24}\end{array}$ & $\begin{array}{l}\text { Electronic waste } \\
\text { leachate }\end{array}$ & 32 & 95 & 2.9 \\
\hline E. coli dLBT $\times 8^{9}$ & $\begin{array}{l}\text { Metal mine } \\
\text { tailing }\end{array}$ & 37 & 90 & 2.4 \\
\hline $\begin{array}{l}\text { Engineered E. } \\
\text { coli }^{25}\end{array}$ & $\begin{array}{l}\text { Geothermal } \\
\text { fluids }\end{array}$ & $9.8 \times 10^{-4}$ & 0.11 & 116 \\
\hline $\begin{array}{l}\text { supported } \\
\text { liquid } \\
\text { membrane }{ }^{19}\end{array}$ & $\begin{array}{l}\text { Coal fly ash } \\
\text { leachate }\end{array}$ & $1.36 \times 10^{-2}$ & 0.53 & 39 \\
\hline $\begin{array}{l}\text { liquid emulsion } \\
\text { membrane }^{19}\end{array}$ & $\begin{array}{l}\text { Coal fly ash } \\
\text { leachate }\end{array}$ & $1.76 \times 10^{-2}$ & 0.37 & 21 \\
\hline
\end{tabular}

aREE purity indicated by Percent REE by mass of total metal.

${ }^{b} \mathrm{REE}$ concentration factor from ratio of REE purity after and before extraction.

\section{References}

(1) Hu, Y.; Drouin, E.; Lariviè, D.; Kleitz, F.; Frédé, F.; Fontaine, F.-G. Highly Efficient and Selective Recovery of Rare Earth Elements Using Mesoporous Silica Functionalized by Preorganized Chelating Ligands. ACS Appl. Mater. Interfaces. 2017, 9(44), 38584-38593.

(2) Noack, C. W.; Perkins, K. M.; Callura, J. C.; Washburn, N. R.; Dzombak, D. A.; Karamalidis, A. K. Effects of Ligand Chemistry and Geometry on Rare Earth Element Partitioning from Saline Solutions to Functionalized Adsorbents. ACS Sustain. Chem. Eng. 2016, 4 (11), 6115-6124.

(3) Callura, J. C.; Perkins, K. M.; Baltrus, J. P.; Washburn, N. R.; Dzombak, D. A.; Karamalidis, A. K. Adsorption Kinetics, Thermodynamics, and Isotherm Studies for Functionalized Lanthanide-Chelating Resins. J. Colloid Interface Sci. 2019, 557, 465-477.

(4) Qi, X. H.; Du, K. Z.; Feng, M. L.; Gao, Y. J.; Huang, X. Y.; Kanatzidis, M. G. Layered A2Sn3S7·1.25H2O (A = Organic Cation) as Efficient Ion-Exchanger for Rare Earth Element Recovery. Journal of the American Chemical Society. 2017, pp 4314-4317.

(5) Li, R.; Greenchem, /; Zhao, F.; Yin, D.; Tam, K. C.; Sillanpää, M.; Repo, E.; Song, Y.; Samia, C.; Hammouda, B.; et al. Polyethylenimine-Cross-Linked Cellulose Nanocrystals for Highly Efficient Recovery of Rare Earth Elements from Water and a Mechanism Study. 2017, 19, 4816.

(6) Ramasamy, D. L.; Wojtuś, A.; Repo, E.; Kalliola, S.; Srivastava, V.; Sillanpää, M. Ligand 
Immobilized Novel Hybrid Adsorbents for Rare Earth Elements (REE) Removal from Waste Water: Assessing the Feasibility of Using APTES Functionalized Silica in the Hybridization Process with Chitosan. Chem. Eng. J. 2017, 330, 1370-1379.

(7) Ahmed, I.; Adhikary, K. K.; Lee, Y.-R.; Ho Row, K.; Kang, K.-K.; Ahn, W.-S. Ionic Liquid Entrapped UiO-66: Efficient Adsorbent for Gd3+ Capture from Water. Chem. Eng. J. 2019, 370, 792-799.

(8) Lee, Y. R.; Yu, K.; Ravi, S.; Ahn, W. S. Selective Adsorption of Rare Earth Elements over Functionalized Cr-MIL-101. ACS Appl. Mater. Interfaces 2018, 10 (28), 2391823927.

(9) Park, D. M.; Brewer, A.; Reed, D. W.; Lammers, L. N.; Jiao, Y. Recovery of Rare Earth Elements from Low-Grade Feedstock Leachates Using Engineered Bacteria. Environ. Sci. Technol 2017, 51, 14.

(10) Ahmed, I.; Lee, Y.-R.; Yu, K.; Bhattacharjee, S.; Ahn, W.-S. Gd 3+ Adsorption over Carboxylic-and Amino-Group Dual-Functionalized UiO-66. Ind. Eng. Chem. Res. 2019, 58 (6), 2324-2332.

(11) Wang, M.; Li, X.; Hua, W.; Shen, L.; Yu, X.; Wang, X. Electrospun Poly(Acrylic Acid)/Silica Hydrogel Nanofibers Scaffold for Highly Efficient Adsorption of Lanthanide Ions and Its Photoluminescence Performance. 2016.

(12) Pereao, O.; Laatikainen, K.; Bode-Aluko, C.; Kochnev, I.; Fatoba, O.; Nechaev, A. N.; Petrik, L. Adsorption of Ce3+ and Nd3+ by Diglycolic Acid Functionalised Electrospun Polystyrene Nanofiber from Aqueous Solution. Sep. Purif. Technol. 2020, 233, 116059.

(13) Aguila, B.; Sun, Q.; Perman, J. A.; Earl, L. D.; Abney, C. W.; Elzein, R.; Schlaf, R.; Ma, S. Efficient Mercury Capture Using Functionalized Porous Organic Polymer. Adv. Mater. 2017, 29 (31), 1700665.

(14) Sun, Q.; Aguila, B.; Perman, J.; Earl, L. D.; Abney, C. W.; Cheng, Y.; Wei, H.; Nguyen, N.; Wojtas, L.; Ma, S. Postsynthetically Modified Covalent Organic Frameworks for Efficient and Effective Mercury Removal. J. Am. Chem. Soc. 2017, 139 (7), 2786-2793. Clark, C. E.; Harto, C. B.; Sullivan, J. L.; Wang, M. Q.; EVS), (. Water Use in the Development and Operation of Geothermal Power Plants.; Argonne, IL (United States), 2010.

(16) Maimoni, A. Minerals Recovery from Salton Sea Geothermal Brines: A Literature Review and Proposed Cementation Process. Geothermics 1982, 11 (4), 239-258.

(17) Ayora, C.; Macías, F.; Torres, E.; Lozano, A.; Carrero, S.; Nieto, -Miguel; Pe, R.; Ferna, A.; Castillo-Michel, H. Recovery of Rare Earth Elements and Yttrium from PassiveRemediation Systems of Acid Mine Drainage. Environ. Sci. Technol 2016, 50, 21.

(18) Liu, P.; Huang, R.; Tang, Y. Comprehensive Understandings of Rare Earth Element (REE) Speciation in Coal Fly Ashes and Implication for REE Extractability. Environmental Science and Technology. 2019, pp 5369-5377.

(19) Smith, R. C.; Taggart, R. K.; Hower, J. C.; Wiesner, M. R.; Hsu-Kim, H. Selective Recovery of Rare Earth Elements from Coal Fly Ash Leachates Using Liquid Membrane Processes. Environ. Sci. Technol. 2019, 53 (8), 4490-4499.

(20) Mochizuki, Y.; Tsubouchi, N.; Sugawara, K. Selective Recovery of Rare Earth Elements from Dy Containing NdFeB Magnets by Chlorination. 2013.

(21) Florek, J.; Chalifour, F.; Bilodeau, F.; Larivière, D.; Kleitz, F. Nanostructured Hybrid Materials for the Selective Recovery and Enrichment of Rare Earth Elements. Adv. Funct. Mater. 2014, 24 (18), 2668-2676. 
(22) Callura, J. C.; Perkins, K. M.; Noack, C. W.; Washburn, N. R.; Dzombak, D. A.; Karamalidis, A. K. Selective Adsorption of Rare Earth Elements onto Functionalized Silica Particles. Green Chem. 2018, 20 (7), 1515-1526.

(23) Ramasamy, D. L.; Wojtuś, A.; Repo, E.; Kalliola, S.; Srivastava, V.; Sillanpää, M. Ligand Immobilized Novel Hybrid Adsorbents for Rare Earth Elements (REE) Removal from Waste Water: Assessing the Feasibility of Using APTES Functionalized Silica in the Hybridization Process with Chitosan. Chem. Eng. J. 2017, 330, 1370-1379.

(24) Brewer, A.; Dohnalkova, A.; Shutthanandan, V.; Kovarik, L.; Chang, E.; Sawvel, A. M.; Mason, H. E.; Reed, D.; Ye, C.; Hynes, W. F.; et al. Microbe Encapsulation for Selective Rare-Earth Recovery from Electronic Waste Leachates. Environ. Sci. Technol 2019, 53, $13888-13897$.

(25) Brewer, A.; Chang, E.; Park, D. M.; Kou, T.; Li, Y.; Lammers, L. N.; Jiao, Y. Recovery of Rare Earth Elements from Geothermal Fluids through Bacterial Cell Surface Adsorption. Environ. Sci. Technol. 2019, 53 (13), 7714-7723. 